\title{
Personal Radio-Frequency Exposimeters in Indoor Diffuse Environments: Measurement and Simulation
}

\author{
Reza Aminzadeh $^{1 *}$, Arno Thielens ${ }^{1}$, Aliou Bamba ${ }^{1,2}$, Lamine Kone ${ }^{2}$, Davy Paul Gaillot ${ }^{2}$, \\ Martine Lienard ${ }^{2}$, Luc Martens ${ }^{1}$, and Wout Joseph ${ }^{1}$ \\ ${ }^{1}$ Department of information technology, Ghent University/iMinds, \\ Gaston Crommenlaan 8, B-9050, Belgium, \\ ${ }^{2}$ Group TELICE, IEMN, University of Lille, France \\ *email: reza.aminzadeh@intec.ugent.be
}

\begin{abstract}
The response of personal exposimeters (PEMs) is studied under diffuse field exposure in indoor environments. A numerical model and a setup for on-body calibration measurements in a reverberation chamber (RC) is proposed for 8975500 MHz. The proposed numerical simulations are in good agreement with measurements. A difference around $2 \mathrm{~dB}$ between their $50 \%$ prediction intervals is observed.
\end{abstract}

Index Terms-diffuse fields, indoor environments, human exposure, personal exposimeters.

\section{INTRODUCTION}

Current technological advancement is accompanied by an increasing number of devices that use various radio-frequency (RF) electromagnetic (EM) sources. In recent years, many researchers have studied EM radiation from several RF sources in real environments [1]-[3]. Personal exposimeters (PEMs) are typically used in these studies to assess exposure to RF EM radiation using the relevant protocols (cf. [1], [4]).

Previous studies (for example [5]) show that PEMs are faced with measurement uncertainties caused by the presence of the human body, because PEMs are calibrated in free-space while they are used on body. This results in deviations of the measured fields from the actual incident fields for which reference levels exist [6].

People spend most of their time indoor and are exposed to EM radiation continuously. According to the room electromagnetics' theory [7], the total power in an indoor environment is composed of specular and diffuse multipath components (DMC), which result from coherent and non-coherent reflections (multiple sets of diffracted waves), respectively. The contribution of DMC to the total power may increase up to $95 \%$ [8]. Therefore, it is necessary to assess the exposure of humans to DMC (in indoor environments). In the previous studies on personal exposimeters, the PEMs' response in diffuse fields is not addressed yet. Currently, the existing calibration procedures are executed in an anechoic chamber. This calibration is studied for specular exposure, but it is uncertain whether this can be used under exposure to DMC.

In this paper, we report on-body calibration measurements and simulations of the response of a PEM, under diffuse field exposure i.e., in a reverberation room. We will compare the results of these measurements with a calibration measurement in an anechoic room. The methodology is described in Section II. Section III presents the results. Finally, Section IV concludes the paper.

\section{Methodology}

The goal of this study is to assess the incident electric fields corrected for the effect of human body using PEMs in indoor environments. A PEM of type EME SPY140 (Satimo, Brest, France) with maximum sample rate of $0.25 \mathrm{~Hz}$ is used. The frequency bands of this PEM are listed in Table I (Lower and upper LTE bands and UMTS/HSPA are not measured by the PEM). The detection limit of the PEM depends on the frequency band and is between 0.005 to $0.02 \mathrm{~V} / \mathrm{m}$.

TABLE I

THE STUDIED FREQUENCY BANDS USED FOR SIMULATIONS AND MEASUREMENTS.

\begin{tabular}{cccc}
\hline RF signal & Range $(\mathrm{MHz})\left(f_{j}\right)$ & $f_{c}(\mathrm{MHz})$ & $L(\mathrm{~mm})$ \\
\hline TV & $470-862$ & 666 & 205.7 \\
Lower LTE-1 $^{*}$ & $791-821$ & 806 & 168.4 \\
Lower LTE-2* $^{*}$ & $832-862$ & 847 & 160.3 \\
GSM 900-UL & $880-915$ & 897 & 151 \\
GSM 900-DL & $925-960$ & 950 & 142.6 \\
GSM1800-UL & $1710-1785$ & 1748 & 73.7 \\
GSM1800-DL & $1805-1880$ & 1843 & 69.9 \\
DECT & $1880-1900$ & 1890 & 67.4 \\
UMTS/HSPA & $1900-1920$ & 1910 & 66.7 \\
UMTS-UL & $1920-1980$ & 1950 & 65.3 \\
UMTS-DL & $2110-2170$ & 2140 & 59.1 \\
WiFi-2G & $2400-2483.5$ & 2450 & 50.5 \\
Upper LTE* & $2500-2570$ & 2600 & 47.2 \\
& $2575-2690$ & & \\
WIMAX & $3400-3600$ & 3500 & 33.8 \\
WiFi-5G & $5150-5875$ & 5500 & 21.2 \\
\hline
\end{tabular}

* Only used for simulations: not in the range of PEM

$f_{c}$ is the center frequency and $L$ the optimized length of the dipole.

\section{A. Numerical Simulations}

To study measurement of PEM on body, the finite-difference time-domain (FDTD) simulations are performed using the SEMCAD-X simulation platform [9]. The PEM is modelled numerically as a dipole. In order to estimate what a PEM will measure in diffuse fields, we determine numerically the distribution of the received RF power on a dipole worn on 
the body in diffuse fields. We consider 14 frequency bands in the range of $666-5500 \mathrm{MHz}$ (from $\mathrm{TV}$ up to WiFi $5 \mathrm{GHz}$ in Belgium [10], see Table I). The location of the PEM on the body is also studied since it can affect its measurements. Fig. 1 shows the details of the proposed numerical model for simulations. For each frequency band, a dipole antenna is placed next to a heterogeneous (81 tissues) virtual family male (VFM) body model [11] with body mass index (BMI) of $22.3 \mathrm{~kg} / \mathrm{m}^{2}$ (see Fig.1(a)). The dielectric properties used for this model are taken from [12] and a maximum grid step of $1.5 \mathrm{~mm}$ is used to resolve the skin of the model, appropriately. The front and back of the torso are chosen for mounting the PEM since in reality, the measurement of a PEM at these locations are less affected by the user's movements. The distance between the dipole and the numerical body model is $19 \mathrm{~mm}$ (half of the width of the EME SPY140).

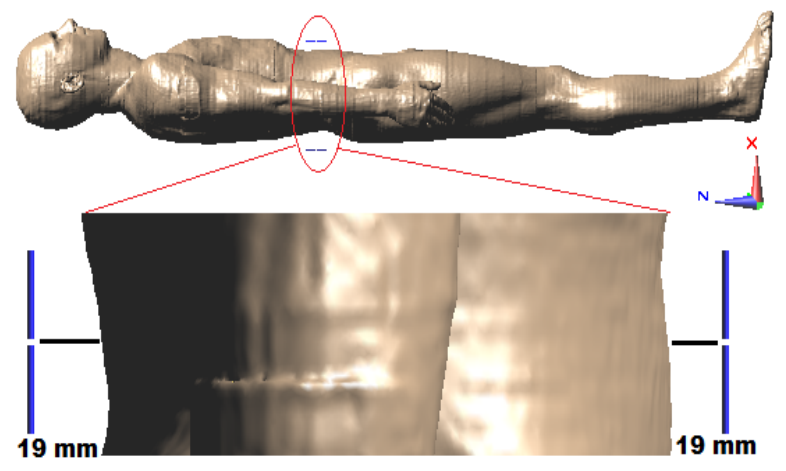

(a)

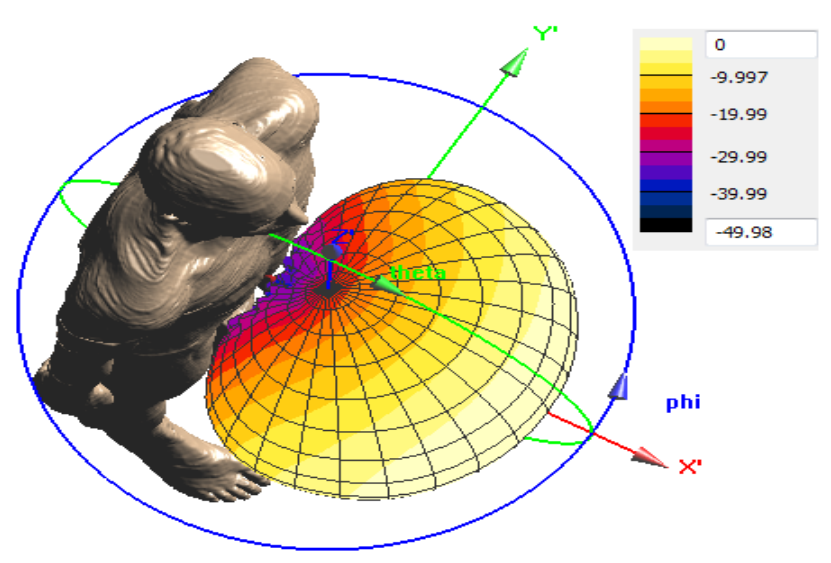

(b)

Fig. 1. The proposed model for numerical simulations. (a) Location of the optimized dipole on the front and the back of the VFM. (b) 3D directivity of the dipole optimized for WiFi $2 \mathrm{GHz}$. (The dipole has a total efficiency of $64.3 \%$ and a return loss equal to $27.7 \mathrm{~dB}$ at $2450 \mathrm{MHz}$, in presence of the body. The directivity is scaled to the maximum directivity in $\mathrm{dB}$.

Firstly, a dipole antenna is designed and optimized at a separation of $19 \mathrm{~mm}$ from a lossy homogeneous phantom $\left(40 \times 20 \times 15 \mathrm{~cm}^{3}\right)$ with dielectric properties of muscle at each center frequency [12] to ensure that the antenna has a return loss lower than $-10 \mathrm{~dB}$. The optimized length of the antenna
$(L)$ is in good agreement with the results of [13] $(0.47 \lambda$ for the dipoles near a body phantom at $2.4 \mathrm{GHz}$ ). These values are listed in Table I. Secondly, in order to simulate exposure to diffuse fields, FDTD simulations are performed for the VFM equipped with a dipole.

To model diffuse indoor fields, we use the model and distributions of [7]. According to [7] uniform distributions are defined and are listed in Table II. The amplitude of the electric fields is assumed to be $1 \mathrm{~V} / \mathrm{m}$ [7].

TABLE II

PROPOSED PARAMETERS FOR SIMULATION

\begin{tabular}{ccc}
\hline Parameter & distribution & range \\
\hline azimuth angle $(\phi)$ & uniform & {$[0,2 \pi]$} \\
polar angle $(\theta)$ & uniform & {$[0, \pi]$} \\
polarization $(\psi)$ & uniform & {$[0, \pi]$} \\
phase $(\alpha)$ & uniform & {$[0,2 \pi]$} \\
No of plane waves $\left(N_{p w}\right)$ & - & 2000 \\
\hline
\end{tabular}

In order to obtain a distribution of dipoles' received power in indoor diffuse fields, a huge number of FDTD simulations should have to be performed. To avoid this, we use the following method. Simulations are performed at each center frequency for the dipole radiating on the front and on the back of torso as shown in Fig. 1(b). Then the 3D directivity of the dipole is determined on the body.

The on-body aperture $A A_{i}$ of the dipole $i$ (front or back) is determined for two orthogonal polarizations $(\theta$ and $\phi)$ from the directive gain $D_{i}$ of each antenna (for $\phi$ and $\theta$ angles) from numerical simulations as:

$$
A A_{i}(\phi, \theta)=\eta_{\text {rad }}\left(1-\left|S_{11}\right|^{2}\right) D_{i}(\phi, \theta) \frac{\lambda^{2}}{4 \pi}
$$

where $\eta_{\text {rad }}$ and $\left|S_{11}\right|^{2}$ are the radiation efficiency and the power reflection coefficient of the dipole, respectively and $\lambda$ is the wavelength. $A A_{i}(\phi, \theta)$ can be determined for two orthogonal polarizations of the incident electric fields: $\theta$ and $\phi$, which are parallel to the unity vectors $\overline{1}_{\theta}$ and $\overline{1}_{\phi}$. These antenna apertures are denoted $A A_{i}\left(\phi, \theta, 0^{\circ}\right)$ and $A A_{i}\left(\phi, \theta, 90^{\circ}\right)$. The received power $P_{r}$ on an antenna $i$ (front or back) can be determined from its aperture [14]:

$$
P_{r, i}(\phi, \theta, \psi)=A A_{i}(\phi, \theta, \psi) \times S_{i n c}
$$

where $S_{\text {inc }}$ are the incident power density from azimuth $(\phi)$ and polar $(\theta)$ angles with a $\psi$ polarization, respectively. $A A_{i}(\phi, \theta, \psi)$ can be calculated for any polarization angle $\psi$ :

$$
\begin{array}{r}
A A_{i}(\phi, \theta, \psi)=A A_{i}\left(\phi, \theta, 90^{\circ}\right) \cdot \cos ^{2}(\psi)+ \\
A A_{i}\left(\phi, \theta, 0^{\circ}\right) \cdot \sin ^{2}(\psi)
\end{array}
$$

Next, a stochastic approach [15] is applied to combine different single plane waves using sets of multiple plane waves to calculate the received power on the dipole and determine the exposure in realistic environments. For multiple plane waves incident on an antenna, the incident plane waves can interfere with each other. Therefore, the received power on a dipole is not necessarily equal to the sum of the induced powers 
induced by each single plane wave. The received power on each antenna $i$ is obtained as a function of the incident electric fields:

$$
\begin{aligned}
& P_{r, i}=\frac{1}{\left|Z_{i}\right|}\left|\sum_{j=1}^{N_{p w}} A F_{i}\left(\theta_{j}, \phi_{j}, 0^{\circ}\right) \cdot\left(\bar{E}_{i n c, j}\left(\theta_{j}, \phi_{j}, \psi_{j}\right) \cdot \overline{1}_{\theta}\right) \cdot e^{i \alpha_{j}}\right|^{2} \\
& +\frac{1}{\left|Z_{i}\right|}\left|\sum_{j=1}^{N_{p w}} A F_{i}\left(\theta_{j}, \phi_{j}, 90^{\circ}\right) \cdot\left(\bar{E}_{i n c, j}\left(\theta_{j}, \phi_{j}, \psi_{j}\right) \cdot \overline{1}_{\phi}\right) \cdot e^{i \alpha_{j}}\right|^{2}
\end{aligned}
$$

where $\left|Z_{i}\right|$ is the magnitude of the input impedance of the dipole $i, \bar{E}_{i n c, j}\left(\theta_{j}, \phi_{j}, \psi_{j}\right)$ is the incident electric field of plane wave $j$ with azimuth angle $\phi_{j}$, polar angle $\theta_{j}$, polarization $\psi_{j}$, amplitude $\left|\bar{E}_{i n c, j}\right|$, phase $\alpha_{j}$ and $N_{p w}$ is the number of incident plane waves. The antenna factor $A F_{i}\left(\theta_{j}, \phi_{j}, \psi_{j}\right)$ is obtained as:

$$
A F_{i}\left(\theta_{j}, \phi_{j}, \psi_{j}\right)=\sqrt{A A_{i}\left(\theta_{j}, \phi_{j}, \psi_{j}\right) \times \frac{\left|Z_{i}\right|}{377}}
$$

\section{B. On-body calibration measurements}

The on-body calibration consists of two steps and is performed in a reverberation chamber (RC). A RC is a closed metallic cavity in which diffuse fields are uniformly distributed. Fig. 2 shows an illustration of the measurement setup. A metallic stirrer is placed in the chamber to stir the EM waves and rotates from $0^{\circ}$ to $360^{\circ}$ with steps of $1^{\circ}$ during the measurements. A constant power of $10 \mathrm{~mW}$ is delivered to the horn antenna (TX) at each center frequency. Performing measurements in the TV band was not possible due to the dimensions (vs. $\lambda_{T V}$ ) of the RC.

In the first step, the free-space incident electric field is measured using a broadband field meter (NBM-550, NARDA, NY, USA) at different heights $(53-203 \mathrm{~cm})$ from the RC's floor and are averaged over the total measured height. The uniformity of diffuse fields is checked by changing the polarization of the TX and repeating the measurements.

In the second step, a 27-year old male subject with a height of $1.81 \mathrm{~m}$ and a mass of $75 \mathrm{~kg}$ (BMI $\left.22.9 \mathrm{~kg} / \mathrm{m}^{2}\right)$ is positioned in the RC (see Fig. 2) wearing a PEM on the front and back of his torso. The electric field strength is measured by the PEM on his body during one complete rotation of the stirrer. The response $\left(R_{i j}\right)$ is defined for the PEM at location $i$ ( $i=$ front or back) and frequency range $f_{j}$ :

$$
R_{i j}\left(f_{j}\right)=\left(\frac{E_{R M S, i j}^{o n-b o d y}\left(f_{j}\right)}{E_{R M S, i j}^{f r e e}\left(f_{j}\right)}\right)^{2}
$$

where $E_{R M S}^{f r e e}$ and $E_{R M S}^{o n-b o d y}$ are obtained from steps 1 and 2, respectively. The obtained responses are then averaged over the front and the back:

$$
R_{a v g, j}\left(f_{j}\right)=\frac{\left(R_{\text {front }, j}\left(f_{j}\right)+R_{\text {back }, j}\left(f_{j}\right)\right.}{2}
$$

In order to compare the results with previously proposed calibrations, on-body calibration measurements are executed in an anechoic room similar to the above mentioned steps and based on the routines proposed in [16].

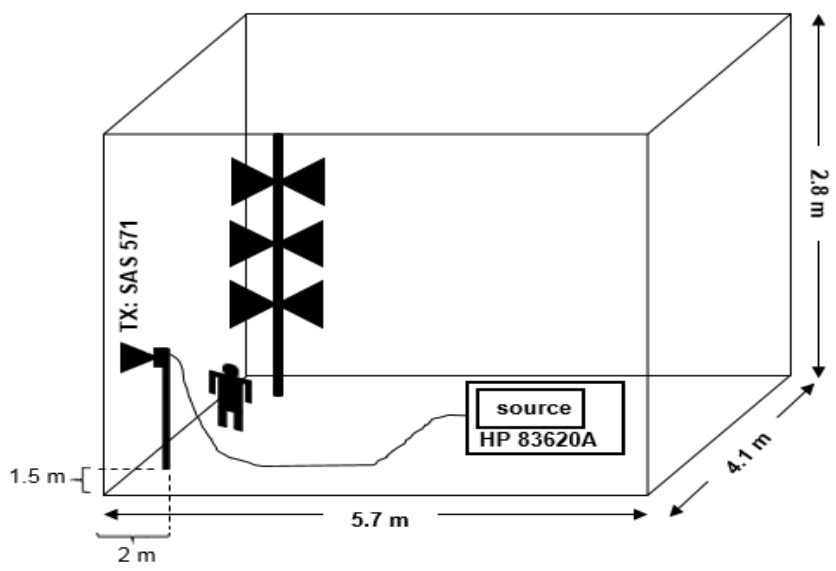

Fig. 2. On-body calibration measurement setup in RC. (Length $=5.7 \mathrm{~m}$; width $=4.1 \mathrm{~m}$; height $=2.8 \mathrm{~m}$ )

\section{RESUlTS AND DISCUSSION}

Fig. 3 shows the median of the simulated received power of the dipole on the front and back of the VFM and for the average over front and back. The best fit for these data is obtained for a second order exponential function $\left(y=a e^{b x}+c e^{d x}\right)$ where $a, b, c$ and $d$ are the coefficients. The received power on the dipole decreases with increasing frequency. This can be explained by equations (2) and (1): $\lambda$ decreases with increasing frequency, which results in a lower $A A_{i}$ and consequently a lower $P_{r, i}$ for a constant $S_{i n c}$.

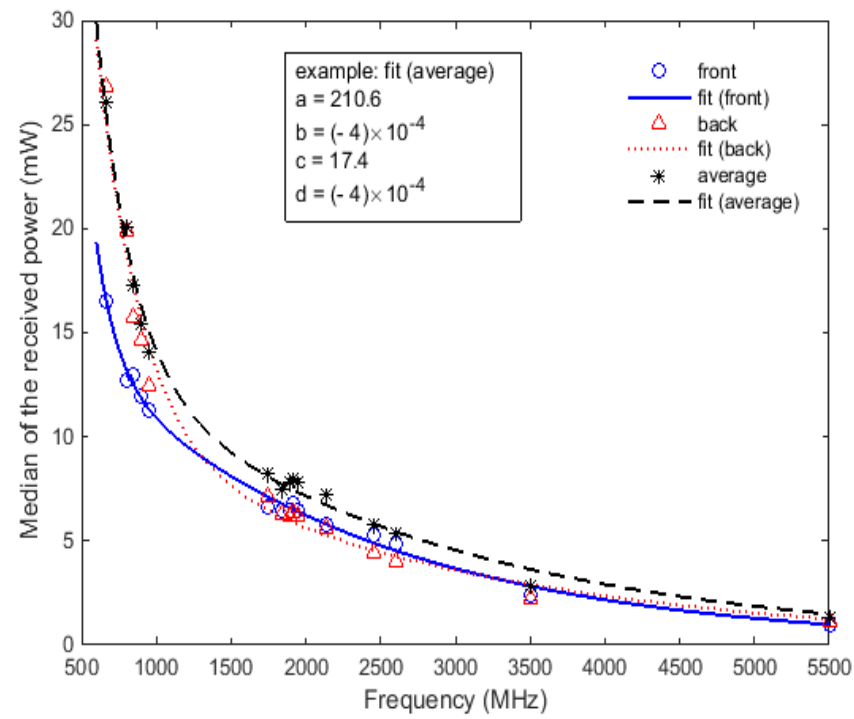

Fig. 3. Fitted curves to the median of the simulated received power on the dipole on the front and back of the body and average over front and back.

Fig. 4(a) shows the $50 \%$ prediction interval $\left(P I_{50}\right)$, i.e., the ratio of $75 \%$ to $25 \%$ percentiles, for the response of the 


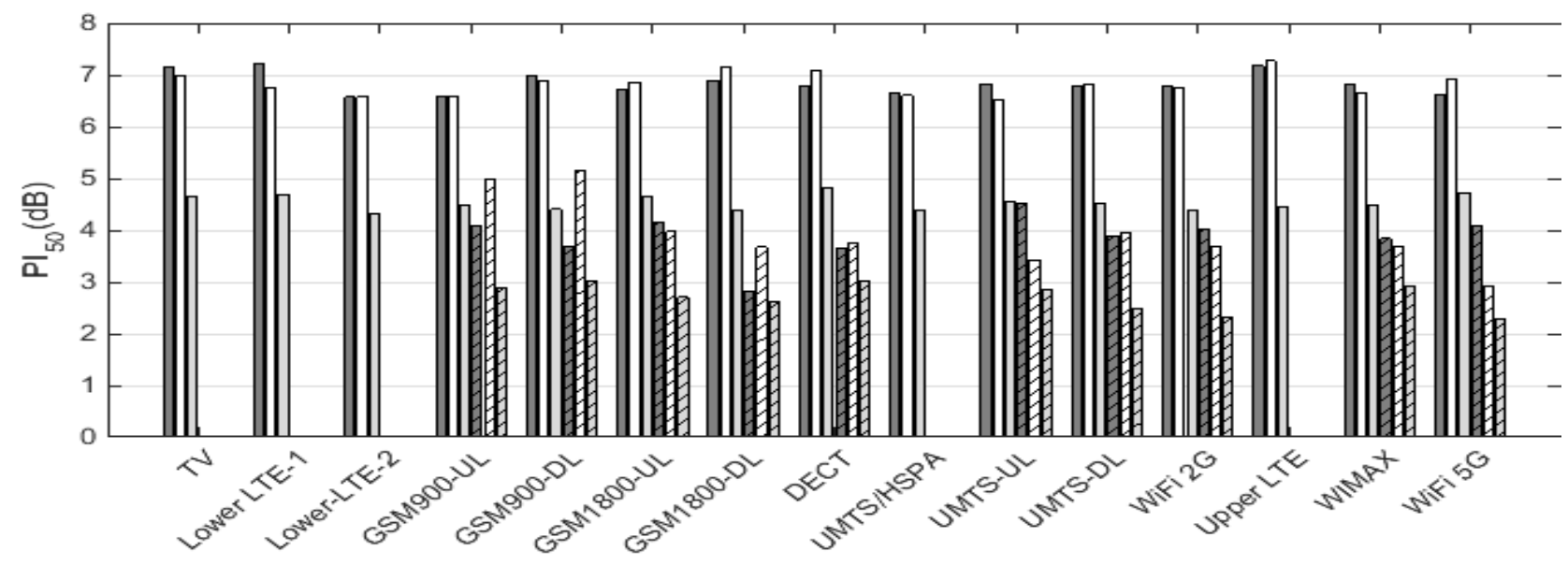

(a) $50 \%$ prediction interval

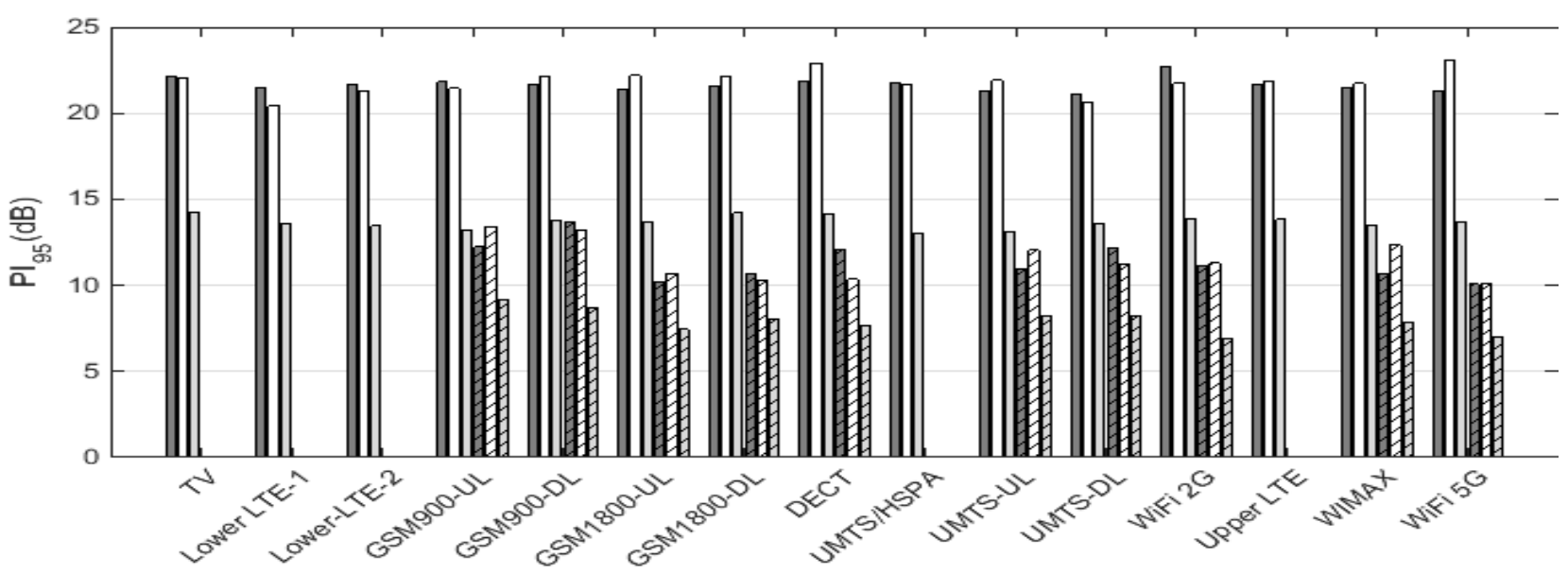

(b) $95 \%$ prediction interval

Fig. 4. The prediction intervals of the response obtained from simulations (bars without pattern) using dipole and measurements (bars with pattern), using PEM, in front (dark gray) and back (white) of the body and average (light gray) over front and back.

simulated dipole and the response of PEM for on-body calibration measurements in RC in the range of $897-5500 \mathrm{MHz}$. Both front and back positions are considered. For simulations (bars without pattern), the values of $P I_{50}$ for the dipole on the front or the back are in the range of 6.5-7.2 dB. Maximum $P I_{50}$ values are found for the lower LTE-1 (dipole on the front) and upper LTE band (dipole on the back). A minimum $P I_{50}$ of $6.5 \mathrm{~dB}$ is obtained from simulations for lower LTE-2 (dipole on the front) and UMTS-UL (dipole on the back). The averaged response for front and back of the VFM results in a $P I_{50}$ of $4.3-4.8 \mathrm{~dB}$. The minimum and maximum $P I_{50}$ values for the average over both positions of the dipole are for lower LTE and DECT, respectively. Averaging over two positions reduces the $P I_{50}$ approximately $2 \mathrm{~dB}$ for all frequency bands.

The results for on-body calibration measurements (bars with pattern) in the RC are as follows: a minimum $P I_{50}$ of $2.8 \mathrm{~dB}$ (GSM1800-DL) for the PEM on the front and $2.9 \mathrm{~dB}(\mathrm{WiFi}-5 \mathrm{G})$ for the PEM on the back is obtained. The maximum values obtained for the $P I_{50}$ are $4.5 \mathrm{~dB}$ (UMTSUL) and 5.1 dB (GSM900-DL) for the PEM on the front and on the back, respectively. For the average over two positions, the $P I_{50}$ is reduced to about $3 \mathrm{~dB}$ for all frequency bands.

The $P I_{50}$ is underestimated by the numerical simulations (approximately $2 \mathrm{~dB}$ ). We attribute this difference to the imperfect modeling of the fields.

Fig. 4(b) shows the $95 \%$ prediction interval $\left(P I_{95}\right)$, i.e., the ratio of $97.5 \%$ to $2.5 \%$ percentiles. For the dipole (simulations, bars without pattern) on the front, it is in the range of $21 \mathrm{~dB}$ (UMTS-DL) to $22.7 \mathrm{~dB}$ (WiFi-2G). For the dipole on the back, it is in the range of $20.4 \mathrm{~dB}$ (lower LTE) to $23.1 \mathrm{~dB}$ (WiFi-5G). For the average over two positions, the $P I_{95}$ is reduced to approximately $13 \mathrm{~dB}$ (UMTS-UL) to 14.2 $\mathrm{dB}$ (TV). Averaging over two positions improved the $P I_{95}$ with approximately $8 \mathrm{~dB}$ for all frequency bands.

For on-body calibration measurements (bars with pattern) in the RC the obtained minimum and maximum $P I_{95}$ values are $10 \mathrm{~dB}$ (WiFi-5G: PEM on the front and on the back) and $13.7 \mathrm{~dB}$ (GSM900-DL: PEM on the front; GSM900-UL: PEM on the back), respectively. For the average over two positions, $P I_{95}$ is reduced to $8 \mathrm{~dB}$ ( $4 \mathrm{~dB}$ improvement). 
The median of the response for the PEM (on the front), as well as the ratio of the median response $(R C / A N)^{-1}$ are listed in Table III. The ratio of the response indicates that using the on-body calibration in anechoic room underestimates the actual exposure in indoor diffuse fields 2-4 times (except WiFi5G) for the PEM on the front. According to the results, the on-body calibration in $\mathrm{RC}$ is advised to assess the exposure in indoor diffuse environments.

TABLE III

RATIO OF THE MEDIAN RESPONSE FOR ON-BODY CALIBRATION MEASUREMENTS FOR RC VERSUS AN FOR THE PEM ON THE FRONT OF SUBJECT'S BODY.

\begin{tabular}{c|ccc}
\hline RF signal & $p_{50}\left(\mathrm{~F}_{R C}\right)$ & $p_{50}\left(\mathrm{~F}_{A N}\right)$ & $(R C / A N)^{-1}(\mathrm{~F})$ \\
\hline GSM 900-UL & 0.06 & 0.13 & 2.1 \\
GSM 900-DL & 0.06 & 0.13 & 2.1 \\
GSM1800-UL & 0.11 & 0.28 & 2.5 \\
GSM1800-DL & 0.09 & 0.28 & 3.1 \\
DECT & 0.008 & 0.02 & 2.5 \\
UMTS-UL & 0.09 & 0.22 & 2.4 \\
UMTS-DL & 0.08 & 0.27 & 3.4 \\
WiFi-2G & 0.12 & 0.5 & 4.1 \\
WIMAX & 0.07 & 0.16 & 2.3 \\
WiFi-5G & 0.13 & 0.13 & 1 \\
\hline
\end{tabular}

$\mathrm{F}=$ front; $(R C / A N)^{-1}$ is the ratio of the median response.

\section{CONCLUSION}

The response of a PEM is studied in indoor diffuse environments. Numerical simulations are performed based on the FDTD method and a stochastic approach is applied to model the response to diffuse fields. On-body calibration measurements are performed in a reverberation chamber under diffuse fields exposure. We show that existing on-body calibration routines that are executed in anechoic chambers underestimate the actual diffuse exposure in indoor environments.

\section{ACKNOWLEDGMENT}

This research was funded by the Research Foundation Flanders (FWO-V) under grant agreement No G003415N.

\section{REFERENCES}

[1] W. Joseph, G. Vermeeren, L. Verloock, M. M. Heredia, and L. Martens, "Characterization of personal RF electromagnetic field exposure and actual absorption for the general public," Health Physics, vol. 95, no. 3, pp. 317-330, 2008.

[2] J. F. Bolte and T. Eikelboom, "Personal radiofrequency electromagnetic field measurements in the Netherlands: Exposure level and variability for everyday activities, times of day and types of area," Environment International, vol. 48, pp. 133 - 142, 2012.

[3] A. Thielens, H. De Clercq, S. Agneessens et al., "Personal distributed exposimeter for radio frequency exposure assessment in real environments," Bioelectromagnetics, vol. 34, no. 7, pp. 563-567, 2013.

[4] M. Röösli, P. Frei, J. Bolte et al., "Conduct of a personal radiofrequency electromagnetic field measurement study: proposed study protocol,' Environ Health, vol. 9, p. 23, 2010.

[5] S. Iskra, R. McKenzie, and I. Cosic, "Factors influencing uncertainty in measurement of electric fields close to the body in personal RF dosimetry," Radiation Protection Dosimetry, vol. 140, no. 1, pp. 25 $33,2010$.
[6] International Commission on Non-ionizing Radiation Protection (ICNIRP), "Guidelines for limiting exposure to time-varying electric, magnetic, and electromagnetic fields (up to $300 \mathrm{GHz}$ )," Health Physics, vol. 74, no. 4, pp. 494-522, 1998.

[7] J. Andersen, J. Nielsen, G. Pedersen, G. Bauch, and M. Herdin, "Room electromagnetics," Antennas and Propagation Magazine, IEEE, vol. 49, no. 2, pp. 27-33, April 2007.

[8] J. Poutanen, J. Salmi, K. Haneda, V. Kolmonen, and P. Vainikainen, "Angular and shadowing characteristics of dense multipath components in indoor radio channels," Antennas and Propagation, IEEE Transactions on, vol. 59, no. 1, pp. 245-253, Jan 2011

[9] SEMCAD-X by SPEAG, SEMCAD X Reference Manual, Schmid \& Partner Engineering AG (SPEAG), February 2012, http://www.speag.com.

[10] Belgian Institute for Postal services and Telecommunications (BIPT), accessed April 2015. [Online]. Available: http://www.bipt.be

[11] A. Christ, W. Kainz, E. G. Hahn et al., "The virtual family-development of surface-based anatomical models of two adults and two children for dosimetric simulations," Physics in Medicine and Biology, vol. 55, no. 2, p. N23, 2010.

[12] C. Gabriel, S. Gabriel, and E. Corthout, "The dielectric properties of biological tissues: I. literature survey," Physics in Medicine and Biology, vol. 41, no. 11, p. 2231, 1996.

[13] L. Roelens, W. Joseph, E. Reusens, G. Vermeeren, and L. Martens, "Characterization of scattering parameters near a flat phantom for wireless body area networks," Electromagnetic Compatibility, IEEE Transactions on, vol. 50, no. 1, pp. 185-193, Feb 2008.

[14] C. A. Balanis, Antenna Theory: Analysis and Design. WileyInterscience, 2005

[15] G. Vermeeren, W. Joseph, C. Olivier, and L. Martens, "Statistical multipath exposure of a human in a realistic electromagnetic environment," Health Phys, vol. 94, no. 4, pp. 345-354, Apr 2008

[16] A. Thielens, S. Agneessens, L. Verloock et al., "On-body calibration and processing for a combination of two radio-frequency personal exposimeters," Radiat Prot Dosimetry, vol. 163, no. 1, pp. 58-69, Jan 2015. 